\title{
Dental Management of Neurofibromatosis Type 1: A Case Report and Literature Review
}

\author{
Fabienne Wotjiuk ${ }^{1}$, Isabelle Hyon ${ }^{2}$, Sylvie Dajean-Trutaud ${ }^{3}$, Zahi Badran $^{4}$, Tony Prud'homme ${ }^{5}$
}

\begin{abstract}
Neurofibromatosis type 1 (NF1) is a clinically heterogeneous neurocutaneous genetic disorder that manifests in the form of coffee-milk spots on the skin, Lish nodules, lentigines on the underarms and on the inguinal region, and neurofibromas. Orofacial manifestations of NF1 are common. Through a review of the literature, bone lesions, orthodontic and dental abnormalities, periodontal manifestations, and caries related to NF1 will be explored. In this study, we present a clinical case of a patient with neurofibroma in the oral cavity and infraocclusion of primary molars, as well as his dental management.
\end{abstract}

Keywords: Dental care, Neurofibromatosis type 1, Oral manifestation.

International Journal of Clinical Pediatric Dentistry (2019): 10.5005/jp-journals-10005-1668

\section{INTRODUCTION}

Neurofibromatosis type 1 (NF1) or von Recklinghausen's disease is a phacomatosis, characterized by an ectodermal tissue abnormality. Its prevalence ranges from $1 / 2500$ to $1 / 3000$, regardless of sex or origin, and is one of the most common genetic diseases. ${ }^{1} \mathrm{NF} 1$ is a genetic disorder, with an autosomal dominant inheritance. It has been found to be linked to the mutation of the NF1 tumor suppressor gene located on chromosome 17, locus q11.2. The latter gene encodes a cytoplasmic protein called neurofibromin, which negatively regulates the proto-oncogene Ras. ${ }^{1,2}$ In the absence of this protein, tumors (most often benign) develop and the bone becomes dysplastic. The mutations of this very large gene are very numerous. Thus, the expression of the disease is very variable, but the penetrance is $100 \%$.

Clinical manifestations of NF1 are extremely variable from one individual to another. They are mostly cutaneous and/or neurological. NF1 patients have a tendency to develop benign or malignant tumors, most often disfiguring and difficult to manage. ${ }^{3}$ Nerve tissue (central nervous system and peripheral nervous system) is primarily affected, but other organs and tissues probably too. $^{2}$

The oral/dental clinician is frequently involved in the patient's care since $72 \%$ of NF1 patients' present oral manifestations. ${ }^{4-7}$

All oral structures can be affected by neurofibromas (single nodules, multiple or plexiform neurofibroma), including the tongue, oral and alveolar mucosa, gingiva, lips, palates, and floor of the mouth. ${ }^{8}$ Nevertheless, the tongue is most often affected. ${ }^{9}$ Neurofibromas can cause tooth movement or mobility, eruption dysfunction, inclusion, or infraocclusion of primary or permanent teeth, mandibular canal widening, hypertrophy of underlying bone structures, gingival hyperplasia, or macroglossia with an increase in fungiform papillae.

The increase in volume of these neurofibromas can cause difficulties in phonation, chewing, swallowing, and facial expression.

This study aims to provide a literature review on the oral expression of NF1 and to present the clinical case of an 8-year-old girl with NF1 involving neurofibroma in the oral cavity.
${ }^{1}$ Prosthetic Dentistry Department, CHU/Université Nantes, France

${ }^{2,3}$ Pediatric Dentistry Department, CHU/Université Nantes, France

${ }^{4}$ Periodontology Department, CHU/Université Nantes, France; Faculty of Dentistry, McGill University, Montreal, Canada

${ }^{5}$ Pediatric Dentistry Department, CHU/Université Nantes, France; UMR 1246 - SPHERE "Methods in Patient-centered Outcomes and Health Research", Université Nantes et Tours, France

Corresponding Author: Tony Prud'homme, Pediatric Dentistry Department, CHU/Université Nantes, France; UMR 1246 - SPHERE "Methods in Patient-centered Outcomes and Health Research", Université Nantes et Tours, France, Phone: +33 240083725, e-mail: tony.prudhomme@univ-nantes.fr

How to cite this article: Wotjiuk F, Hyon I, Dajean-Trutaud S, et al. Dental Management of Neurofibromatosis Type 1: A Case Report and Literature Review. Int J Clin Pediatr Dent 2019;12(6):577-581.

Source of support: Nil

Conflict of interest: None

\section{BACKGROUND}

\section{Maxillary, Mandibular and Temporomandibular Joint Bone Lesions}

In addition to orbital and sphenoidal wings dysplasia, maxillary, mandibular, and temporomandibular joint deformities have been reported in the literature. ${ }^{10}$ An estimated $28 \%$ of the children present radiological abnormalities, specific of NF1 and $36 \%$ present a deformation of the sigmoid notch and the condyle head. ${ }^{10}$ The mandibular lesions can be explained by their similarity with the long bones. Bone lesions, although not found in all patients with NF1, can be explained by the increase of the osteoclastic activity and by local factors such as the presence of tumors. ${ }^{6}$ The relationship between maxillofacial malformations and neurofibromas is unclear, but the most severe malformations are associated with the presence of plexiform neurofibromas. ${ }^{3}$ These cause unilateral jaw malformations and tooth migration. Although neurofibromas are present in primary dentition, development and size of permanent 
teeth are not affected. ${ }^{11}$ In addition, when facial deformities are present from childhood, they scarcely progress in adulthood. The growth potential of plexiform neurofibromas decreases with increasing age. ${ }^{5}$

Also, in patients with NF1, enlargement of the lower alveolar canal is frequently observed. ${ }^{3,5}$

\section{Periodontal Manifestations}

In patients with NF1, high scores of plaque and bleeding indices are often present, as well as an increased clinical attachment loss is high. ${ }^{6}$ However, Bardellini et al. found that children diagnosed with NF1 have higher plaque scores compared to healthy patients. This tendency was not found when comparing gingival bleeding. ${ }^{10}$

\section{Dental Abnormalities}

As with bone lesions, dental abnormalities are strongly associated with plexiform neurofibromas in the territory of the trigeminal nerve. Friedrich et al. reported aberrations in the number of teeth (agenesis or hyperdontia with retained teeth), molar retentions, molar distalization, aplasia of mandibular second molars, increased interdental spaces, and asymmetric jaw on the side reached by a plexiform neurofibroma. ${ }^{3}$ Bardellini et al. ${ }^{10}$ point the presence of taurodontism, agenesis, and enamel dysplasia in patients with NF1. However, there is no statistical significant difference between prevalence in children with NF1 and in patients without NF1.

Craniofacial structures are affected but there are a few studies on the effect of NF1 on dental development. Lammert et al., ${ }^{12}$ in a study based on a retrospective questionnaire, note an early eruption of temporary teeth in patients with NF1. However, this association is not reported in any other study. Thus, for Jääsaari et al., ${ }^{13}$ there is no significant difference in dental age between the control population and patients with NF1.

According to Vinaspuu et al., $34.8 \%$ of women with NF1 had periapical cement dysplasia on mandibular vital teeth that was not present in men or children. ${ }^{14}$

\section{Orthodontic Abnormalities}

From an orthodontic point of view, deformations of alveolar ridges cause complex malocclusions. ${ }^{3}$ Children with NF1 are more likely to have a class III molar and unilateral posterior crossbite. ${ }^{12,13}$ Early orthodontic evaluation should be planned to limit invasive orthodontic treatment. $^{15}$

\section{Caries and NF1}

The sometimes described increase in the prevalence of caries ${ }^{16}$ in children with NF1 is controversial. In a Canadian retrospective study, ${ }^{16}$ Tucker et al. sent a questionnaire on the number of decayed or treated teeth to families with at least 1 child with NF1. Among the 37 siblings analyzed, there are more caries in children with NF1 compared to children without NF1 $(p<0.05)$. After clinical examination, Tsang et al. ${ }^{17}$ and Bardellini et al. ${ }^{10}$ found no significant difference in the prevalence of caries in children with or without NF1. The increase in the number of caries can be explained by the vitamin D deficiency in patients with NF1, ${ }^{18}$ which impacts the amelogenesis, but especially by dental plaque, which can be linked to the delay in the acquisition of motor skills allowing essential for efficient oral cleaning. Indeed, the accumulation of dental plaque associated with poor oral hygiene plays a determining role in the carious prevalence regardless of the general health of the patient. ${ }^{6}$ In contrast, Visnapuu et al. ${ }^{19}$ found in a Finnish population that before the age of 35 , the carious prevalence in patients with NF1 is lower than the control population. The link between caries and neurofibromatosis is therefore not clearly defined in the literature.

\section{Case Description}

\section{Patient Presentation}

We received in consultation, at the Dental Center of Nantes University Hospital, an 8-year-old girl for multifocal dental pain since 1.5 months. The patient's medical history revealed a neurofibromatosis type 1 with hydrocephalus, causing intracranial hypertension. The latter was treated by ventriculoperitoneal bypass. This constitutes a contraindication of the nitrous oxide use. She also presents a glioma with thickening of the nerve and the optic chiasm. She is intellectually and developmentally retarded. This medical history does not allow ambulatory management for the realization of complex, invasive or long care.

\section{Physical Examination}

During the interrogation, the parents reported us a delay of eruption of the permanent teeth. Because of the patient's mental deficiency, pain assessment using an analog visual scale (VAS) is not significant.

Extra-oral examination (Figs 1 to 3 ) revealed a left plexiform orbital and temporal neurofibroma as well as pigmented spots and macules "coffee-milk", without relief, of varying sizes.

At the intra-oral examination (Figs 4 to 6), we noticed the presence of visible plaque, a sign of inadequate oral hygiene. The patient has a left unilateral maxillary gingival hypertrophy, firm, diffuse, and non-inflammatory. The teeth 85555465647516 46 present carious lesions.

We also note the infraocclusion of primary teeth (65 75 85). In addition to these eruption abnormalities, the patient also has dental malpositions and occlusion disorders.

We performed an orthopantomogram to clarify the dental infraocclusion and the positions of absent teeth (Fig. 7).

\section{Dental Management}

The patient received instructions on oral care and dental healthy diet. Zinc oxide eugenol medications were placed on carious teeth. A few weeks later, the patient was treated under general anesthesia. An antibioprohylactic regimen of amoxicillin $(1.25 \mathrm{~g}$

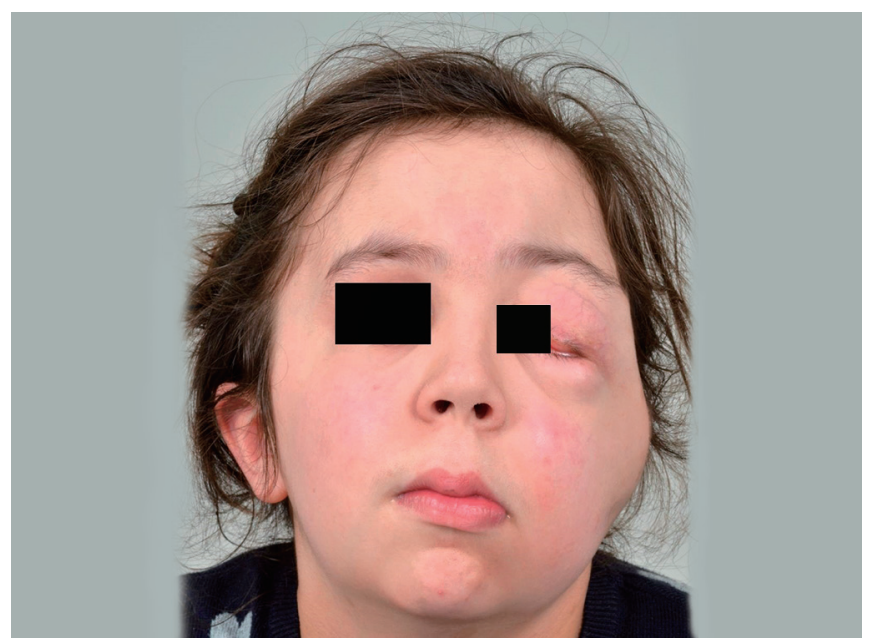

Fig. 1: Frontal exo-oral view 


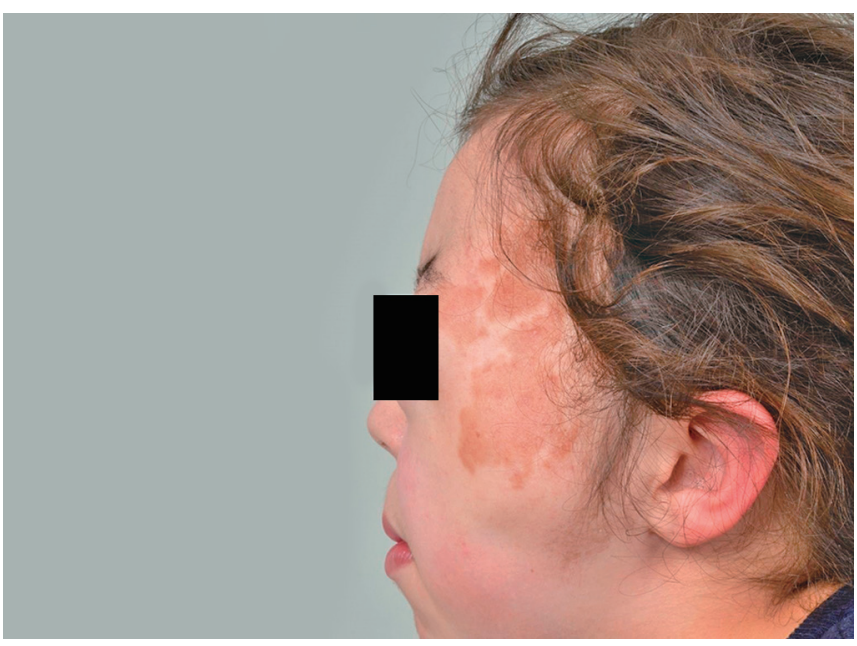

Fig. 2: Left lateral exo-oral view

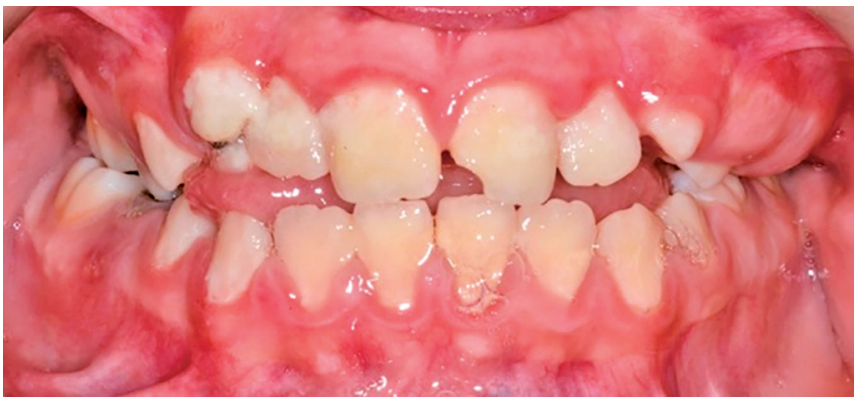

Fig. 4: Initial frontal endo-oral view

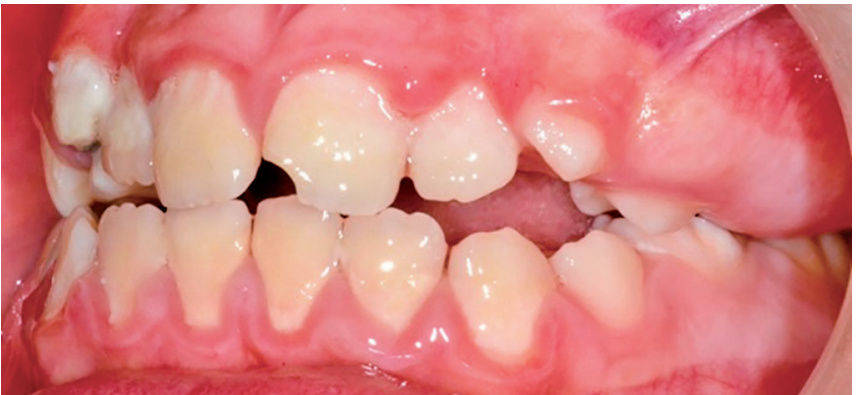

Fig. 6: Initial left lateral endo-oral view

1 hour before intervention) was prescribed. We carried out scaling and root planing and teeth avulsions (85 535565 75). The teeth 64 and 54 exfoliated naturally between the initial consultation and the general anesthesia. After carious lesions removal, restorations were placed in occlusal teeth 46 and 16 . Resin composite was used to restore the 21 mesial angle (loss of substance from traumatic origin).

Owing to the bleeding risk inherent to extractions of the infraocclused primary molars and the fibroid site on left maxillar, various precautions were taken. Local anesthesia of the extraction sites allowed local vasoconstriction. The surgical procedure was the minimally invasive. After completion of the avulsions, haemostatic sponges were placed in the alveolar sockets and we performed sutures using a 3-0 vicryl suture. At the end of the procedure, the hemostasis was checked after local compression (Fig. 8).

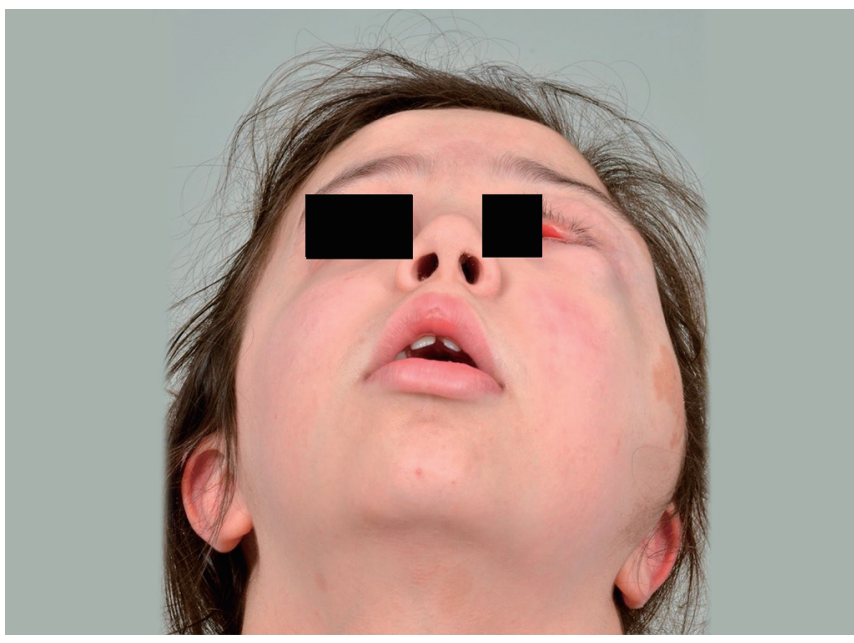

Fig. 3: Underside exo-oral view

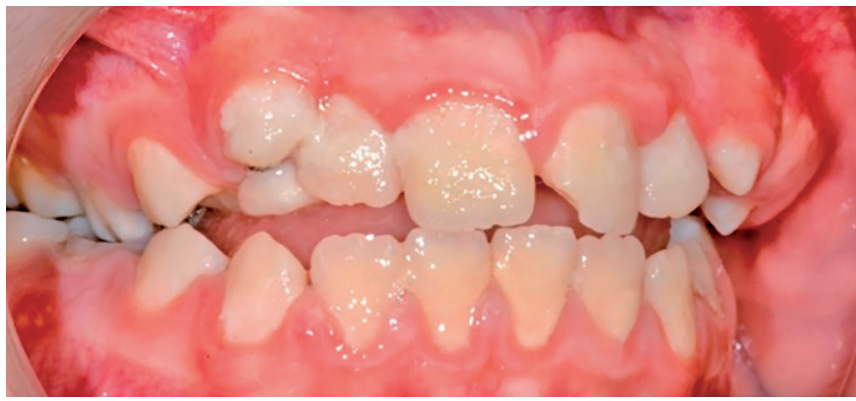

Fig. 5: Initial right lateral endo-oral view

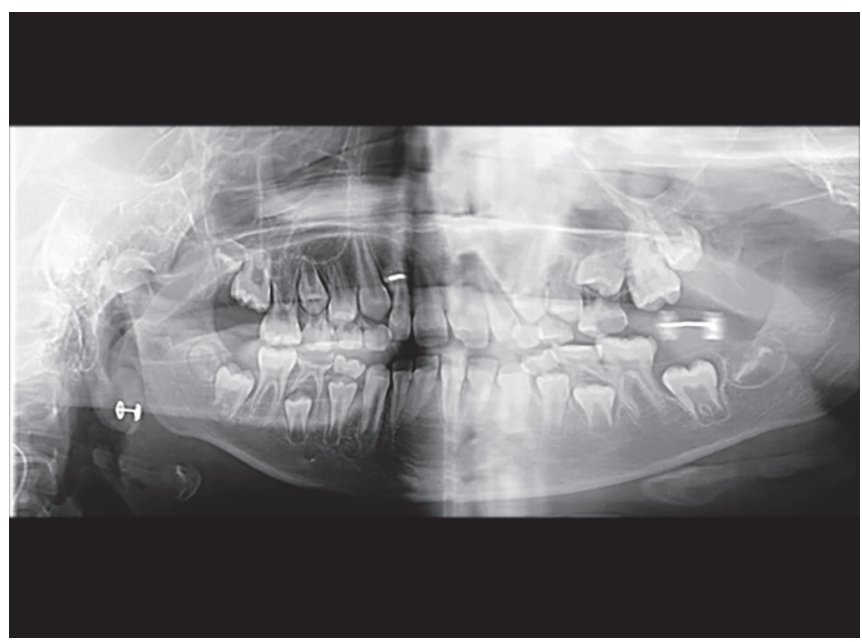

Fig. 7: Initial orthopantomogram

\section{Physical Examination}

At the one-week postoperative followup, regular oral care was recommended to parents. This monitoring will make possible to implement measures of prevention and oral hygiene but also to monitor the stability of the volume and appearance of the fibroid. The resection of the fibroid is not considered for the moment because of the high probability of recurrence and the high risk of bleeding. However, its risk of malignant degeneration must be taken into account. ${ }^{6}$ 


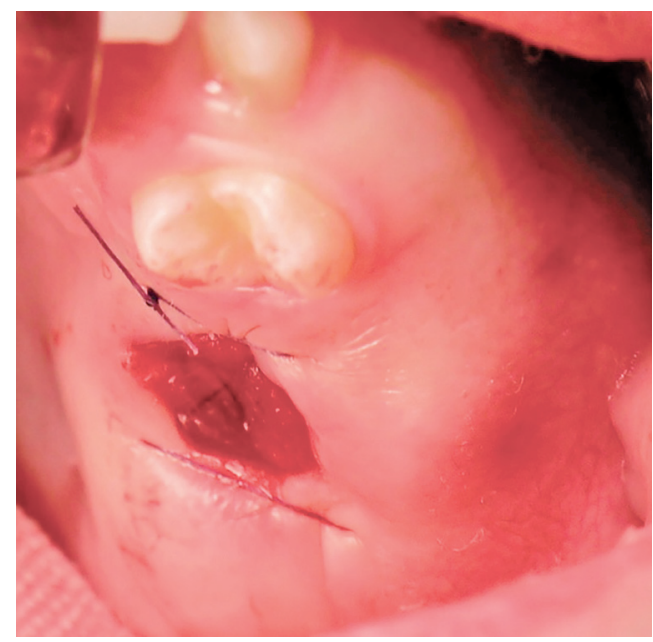

Fig. 8: Postextraction view of the second left primary molar at the plexiform neurofibroma location

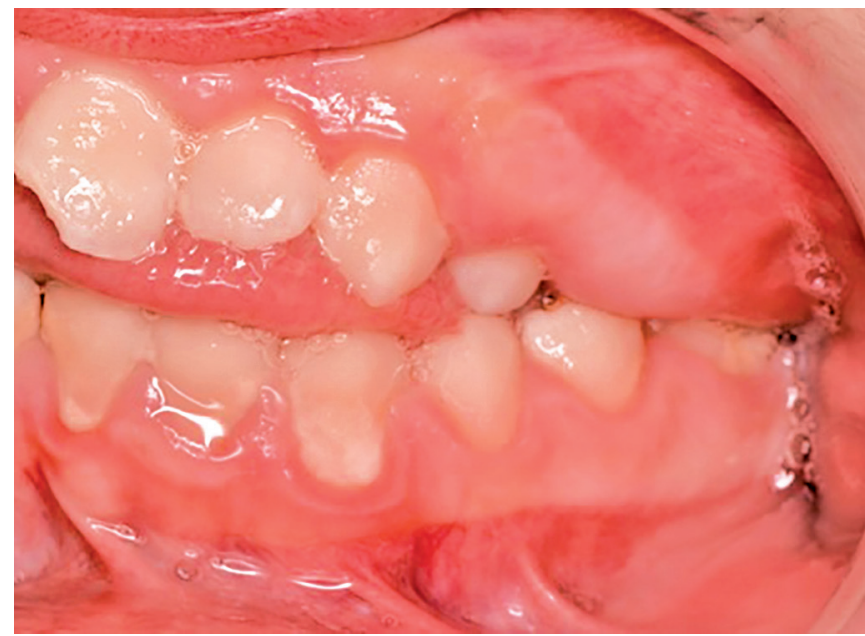

Fig. 10: Left lateral endo-oral view at 2 years of the intervention

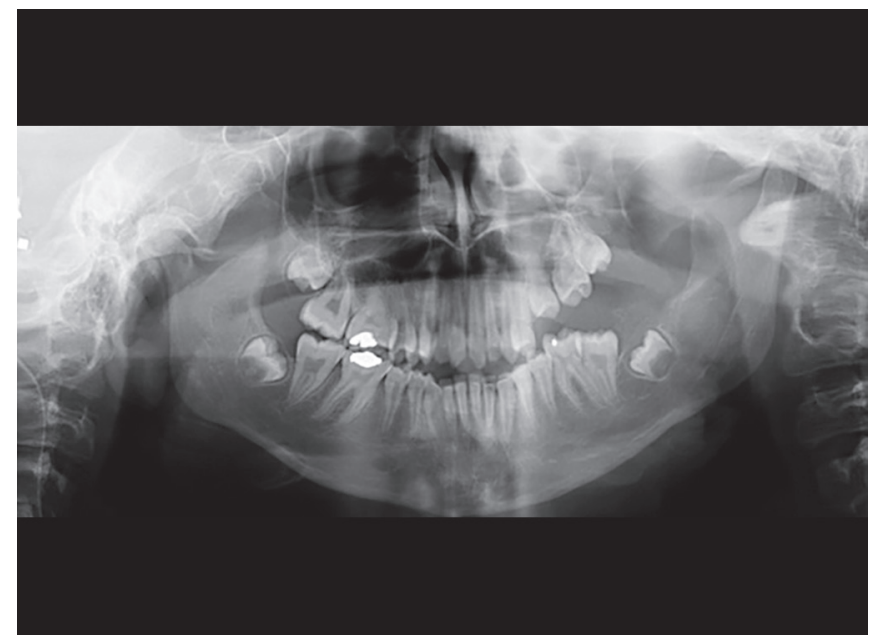

Fig. 12: Orthopantomogram at 2 years of the intervention

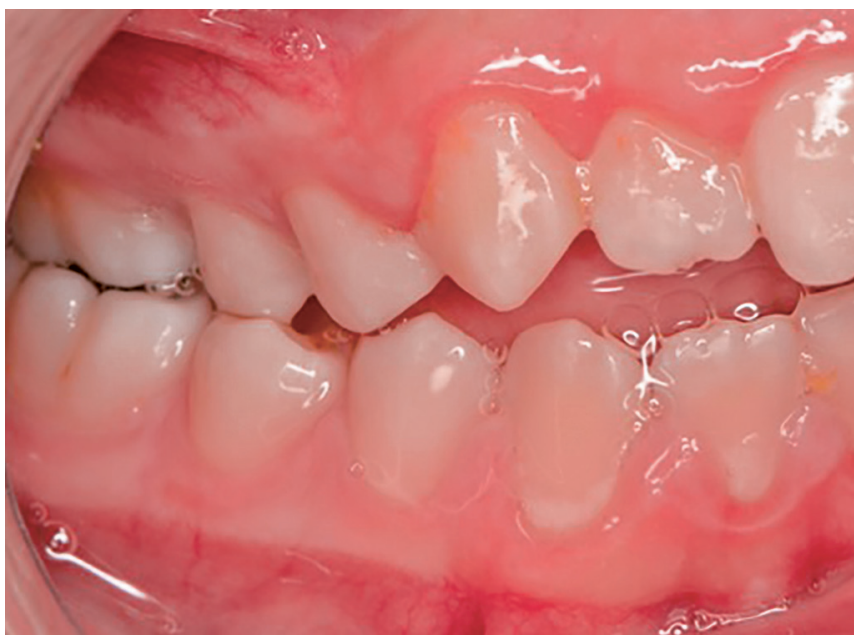

Fig. 9: Right lateral endo-oral view at 2 years of the intervention

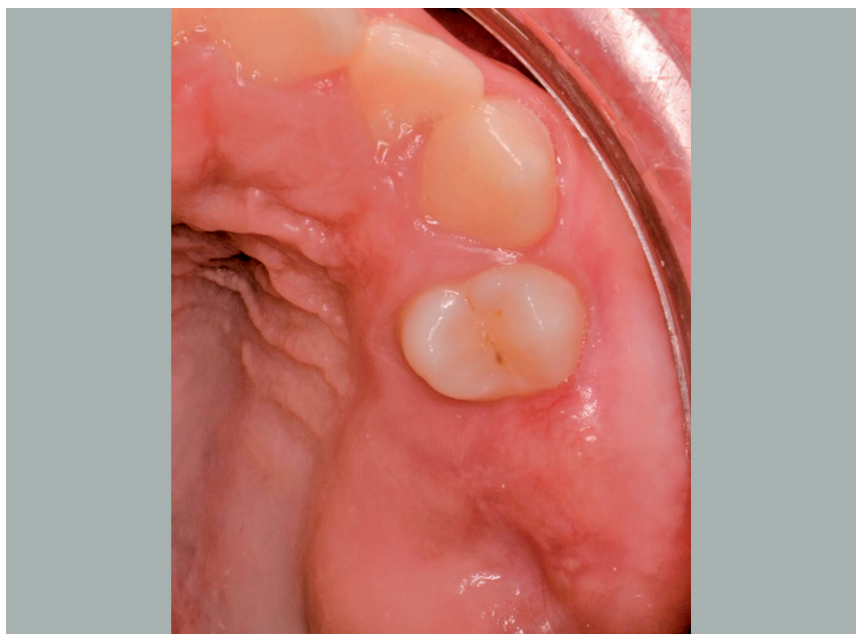

Fig. 11: Occlusal view of the posterior left maxillary at 2 years of intervention

At the 2-year follow-up examination, the patient was reviewed regularly every 6 months. Oral hygiene remains perfectible. The evolution of the premolars was satisfactory following the performed extractions, except in sector 2, where the neurofibroma prevents the evolution of 25 and 16 (Figs 9 to 12). The resection of the fibroid is still not considered.

\section{Discussion}

NF1 has a large polymorphism. All described lesions are not found in all patients. In the 1950 s, doctors claimed that $4-7 \%$ of patients with NF1 had oral manifestations. In the 80 s, thanks to radiographic analysis, different authors showed that this proportion is much higher and estimated to be $66-72 \% .^{8,9}$

It is difficult to predict the course of the disease. Oral manifestations may be present in childhood even if the main complication of the disease (neurofibromas) is not yet developed. ${ }^{10}$

Infraocclusion of teeth are common in NF1 field. This frequently requires the avulsion of the primary teeth concerned. In patients 
with NF1, the operative risk is mainly based on hemorrhagic complications, when extractions take place in plexiform neurofibromas field, which are sometimes hypervascular lesions. Dysplastic vessels are responsible for important bleeding. The French High Authority of Health, via the National Protocol of Care publication, recommends, in these patients, a hemostasis assessment before any dental surgical procedure as well as a rigorous hemostasis with adapted material and technique during the intervention.

\section{Conclusion}

More than $70 \%$ of patients with NF1 have oral complications. ${ }^{6}$ It is therefore important for the dentist to know them and know how to take care of them. In these patients, special attention should be paid to oral hygiene education and regular dental followup. Thus, the dentist will be part of the multidisciplinary teams that take care of this complex neurocutaneous pathology. Through this work and in particular the clinical case presenting extractions in field of plexiform neurofibromas, we hope to help in the care of these patients.

\section{References}

1. Hirbe AC, Gutmann DH. Neurofibromatosis type I: a multidisciplinary approach to care. Lancet Neurol 2014 Aug;13(8):834-843. DOI: 10.1016/S1474-4422(14)70063-8.

2. Ferner RE. Neurofibromatosis 1. Eur J Hum Genet EJHG 2007 Feb;15(2):131-138. DOI: 10.1038/sj.ejhg.5201676.

3. Friedrich RE, Giese M, et al. Jaw malformations plus displacement and numerical aberrations of teeth in neurofibromatosis type I: a descriptive analysis of 48 patients based on panoramic radiographs and oral findings. J Cranio-Maxillo-fac Surg Off Publ Eur Assoc Cranio-Maxillo-fac Surg 2003 Feb;31(1):1-9. DOI: 10.1016/S10105182(02)00160-9.

4. Cunha KSG, Barboza EP, et al. Neurofibromatosis type I with periodontal manifestation. A case report and literature review. $\mathrm{Br}$ Dent J 2004 Apr 24;196(8):457-460. DOI: 10.1038/sj.bdj.4811175.

5. Visnapuu V, Peltonen S, et al. Radiographic findings in the jaws of patients with neurofibromatosis 1. J Oral Maxillofac Surg Off J Am Assoc Oral Maxillofac Surg 2012 Jun;70(6):1351-1357. DOI: 10.1016/ j.joms.2011.06.204.
6. Javed F, Ramalingam S, et al. Oral manifestations in patients with neurofibromatosis type I: a comprehensive literature review. Crit Rev Oncol Hematol 2014 Aug;91(2):123-129. DOI: 10.1016/ j.critrevonc.2014.02.007.

7. Geist JR, Gander DL, et al. Oral manifestations of neurofibromatosis types I and II. Oral Surg Oral Med Oral Pathol 1992 Mar;73(3):376-382. DOI: 10.1016/0030-4220(92)90139-H.

8. D'Ambrosio JA, Langlais RP, et al. Jaw and skull changes in neurofibromatosis. Oral Surg Oral Med Oral Pathol 1988 Sep;66(3): 391-396. DOI: 10.1016/0030-4220(88)90252-6.

9. Shapiro SD, Abramovitch K, et al. Neurofibromatosis: oral and radiographic manifestations. Oral Surg Oral Med Oral Pathol 1984 Oct;58(4):493-498. DOI: 10.1016/0030-4220(84)90350-5.

10. Bardellini $E$, Amadori $F$, et al. Oral findings in 50 children with neurofibromatosis type I. A case control study. Eur J Paediatr. Dent Off J Eur Acad Paediatr Dent 2011 Dec;12(4):256-260.

11. Friedrich RE, Giese M, et al. Size of tooth crowns and position of teeth concerning the extension of facial plexiform neurofibroma in patients with neurofibromatosis type I. Anticancer Res 2012 May;32(5):2207-2214.

12. Lammert M, Friedrich RE, et al. Early primary tooth eruption in neurofibromatosis 1 individuals. Eur J Oral Sci 2007 Oct;115(5): 425-426. DOI: 10.1111/j.1600-0722.2007.00474.x.

13. Jääsaari $P$, Visnapuu $V$, et al. Dental age in patients with neurofibromatosis 1. Eur J Oral Sci 2012 Dec;120(6):549-552. DOI: 10.1111/j.1600-0722.2012.01000.x.

14. Visnapuu V, Peltonen S, et al. Periapical cemental dysplasia is common in women with NF1. Eur J Med Genet 2007 Aug;50(4):274-280. DOI: 10.1016/j.ejmg.2007.04.001.

15. Bardellini E, Tonni I, et al. Occlusal traits in children with neurofibromatosis type I. Orthod Craniofac Res 2016 Feb;19(1):46-53. DOI: $10.1111 / o c r .12108$.

16. Tucker $\mathrm{T}$, Birch $\mathrm{P}$, et al. Increased dental caries in people with neurofibromatosis 1. Clin Genet 2007 Dec;72(6):524-527. DOI: 10.1111/j.1399-0004.2007.00886.x.

17. Tsang ES, Birch $P$, et al. Prevalence of dental caries in children with neurofibromatosis 1. Clin Oral Investig 2010 Aug;14(4):479-480. DOI: 10.1007/s00784-009-0361-6, author reply 480.

18. Lammert M, Friedman JM, et al. Vitamin D deficiency associated with number of neurofibromas in neurofibromatosis 1. J Med Genet 2006 Oct;43(10):810-813. DOI: 10.1136/jmg.2006.041095.

19. Visnapuu V, Pienihäkkinen K, et al. Neurofibromatosis 1 and dental caries. Clin Oral Investig 2011 Feb;15(1):119-121. DOI: 10.1007/s00784009-0341-x. 